\title{
The universe formation by a space reduction cascade with random initial parameters
}

\author{
S.G. RubiN AND A.S. Zingen \\ National Research Nuclear University "MEPhI", \\ Kashirskoe sh. 31, Moscow, 115409 Russia
}

\begin{abstract}
In this paper we discuss the creation of our universe using the idea of extra dimensions. The initial, multidimensional Lagrangian contains only metric tensor. We have found many sets of the numerical values of the Lagrangian parameters corresponding to the observed low-energy physics of our universe. Different initial parameters can lead to the same values of fundamental constants by the appropriate choice of a dimensional reduction cascade. This result diminishes the significance of the search for the 'unique' initial Lagrangian. We also have obtained a large number of low-energy vacua, which is known as a 'landscape' in the string theory.
\end{abstract}

PACS numbers: 04.50.-h, 04.50.Cd

November 20, 2018

\footnotetext{
$*<$ sergeirubin@list.ru $>$

$\dagger<$ zinger.alexey@gmail.com $>$
} 


\section{Introduction}

In this paper we investigate the problems of an emergence of fundamental constants as well as other parameters at an early stage of the Universe evolution. It is well known that the range of admissible parameters must be extremely narrow (so-called fine tuning of parameters) for such complex structures as our Universe to exist, which is difficult to explain. Extensive literature is devoted to the discussion of this problem 11. One way of solving it is based on the assumption of the multiplicity of universes with different properties [2], 3], 4]. Rich possibilities for justifying this assumption are contained in the idea of multidimensionality of the space. The number of extra dimensions has long been a subject of debate. For instance, the Kaluza-Klein model originally contained one extra dimension. Now infinite-dimensional spaces [5] and even variabledimensional spaces [6] are being discussed.

The idea of the multiplicity of universes [7] usually implies an existence of the initial Lagrangian with specific parameters and having potential density with numerous local minima. Each of these minima corresponds to a certain low-energy effective theory with its own unique set of parameters. Which of the minima we get to depends on the initial conditions. Therefore, observed lowenergy physics depends not only on the initial parameters of the Lagrangian, but also on the initial metric tensor of the created space-time.

This idea is usually developed within the framework of the string theory [13], which includes, among other assumptions, an existence of extra dimensions. In this paper we develop a purely geometrical approach [8], postulating only the existence of additional dimensions. We use the cascade reduction mechanism, introduced in [9], to explain the fine tuning of the parameters in our universe without involving the string theory assumptions other than existence of extra dimensions.

The essence of our idea is as follows. Consider a space of multiple dimensions. Because of quantum fluctuations in some of its regions, a geometry of the direct product of two subspaces may arise. Suppose the curvature of one of the subspaces significantly exceeds that of the other; let us refer to the former subspace as the extra one and to the latter as the main subspace. Quantum fluctuations in some region of a newly formed main subspace similarly divide it into direct product of two subspaces. Considering further divisions we arrive at a "chain" of partitions of the space. Every such partition we shall call a reduction of the space. Multiple consecutive reductions we shall call a cascade of reductions.

Thus a cascade of reductions consists of several steps, reducing effective dimensionality of the space. It will be shown that every step of a cascade changes parameters of Lagrangian. Therefore, by choosing different cascades we can obtain different final Lagranigians starting from a fixed initial parameters. Each Lagrangian corresponds to universes with distinct properties. It is this possibility that is usually associated with the concepts of the landscape - numerous low-energy vacua. Our goal is, in a sense, the opposite one: we try to assess the set of all the parameters of the initial Lagrangian leading to the observed 
fundamental constants.

In this paper we show that there are numerous initial Lagrangians leading to the observable physics. It will be shown that the low-energy physics depends not only on the initial parameters, but also on the properties of the compact spaces in particular cascade. Therefore a variation of initial parameters may be compensated by an appropriate variation of the properties of the cascade, leaving the low-energy physics unchanged. This diminishes the importance of the search for the "unique" Lagrangian of the Theory of Everything.

We also discuss the relationships between the parameters of the initial Lagrangian and fundamental constants $\hbar$ and $G$, determined in low-energy experiments.

\section{Reduction cascades}

\subsection{Main idea}

Let us discuss the main idea in detail. Consider a $D$-dimensional space $M_{D}$. Because of its metric fluctuations, new spatial regions with various geometries are constantly born within it. We'll be interested in regions with the direct product geometry of the form

$$
U_{1}=T \otimes M_{d_{1}} \otimes M_{D_{1}}, \quad U_{1} \subset M_{D}
$$

Here $T$ is the timelike direction, $M_{d_{1}}$ is a compact space of $d_{1}$ dimensions, $M_{D_{1}}$ is the main space, whose metric fluctuations are studied at the next step of the cascade. We emphasize that geometry is changed not in the whole space $M_{D}$, but only in its small region $U_{1}$. All physical processes are considered from the viewpoint of an observer located inside the subspace $M_{D_{1}}$.

Let us limit our consideration to quantum fluctuations satisfying the following relationship for Ricci scalars in subspaces $M_{d_{1}}$ and $M_{D_{1}}$

$$
R_{d_{1}}>R_{D_{1}} .
$$

In case of the simplest geometries, the bigger the curvature of the space, the less is its volume. This is the type of geometries we'll be studying. But the less the volume of the system, the faster are the relaxation processes in it (see discussion in [9]). Therefore, due to condition (2), processes in $M_{d_{1}}$ advance much faster than those in $M_{D_{1}}$. In what follows we discuss the conditions to stabilize the volume of $M_{d_{1}}$ and its geometry. Theory becomes effectively $D_{1^{-}}$ dimensional, with $d_{1}$ compact extra dimensions. The parameters of the initial $D$-dimensional Lagrangian are renormalized, with their new values depending on the properties of the compact extra space $M_{d_{1}}$.

Described above is the first step of the cascade. The following steps are similar: due to the metric fluctuations in some volume of the newly formed space $M_{D_{1}}$, there arises a new geometry

$$
U_{2}=T \otimes M_{d_{2}} \otimes M_{D_{2}}, \quad U_{2} \subset T \otimes M_{D_{1}}
$$


resulting in segregation of another compact space $M_{d_{2}}$. Ricci scalars of both new subspaces should satisfy the condition, analogous to (2):

$$
R_{d_{2}}>>R_{D_{2}}
$$

Parameters of the reduced Lagrangian are renormalized once more; their values become dependent on the properties of the compact extra space $M_{d_{2}}$.

Cascades differ from each other by the properties of its compact subspaces: their volume, topology and geometry.

Each reduction consists of two stages: first is the quantum formation of the space of the form (11); second is the classical evolution of this space, which results in stabilization of the compact extra space $M_{d_{i}}$.

\subsection{The first step of the cascade}

\subsubsection{Quantum formation of the space}

In this section we discuss the probability of a quantum formation of geometries we are interested in. Let us consider a $D$-dimensional space. Quantum fluctuations in small regions of this subspace create subspaces of the form $M_{d_{1}} \otimes M_{D_{1}}$.

The transitions with a change in the geometry are conveniently described in terms of the path integral technique [22, 25]. For this purpose, the superspace $\mathcal{M}_{D}=\left(M_{D} ; g_{i j}\right)$ is defined as a set of metrics $g_{i j}$ in the space $M_{D}$ to within diffeomorphisms. On a spacelike section $\Sigma$ we introduce the metric $h_{i j}$ (for details, see [26]) and define the space of all Riemannian $(D-1)$ metrics in the form

$$
\operatorname{Riem}(\Sigma)=\left\{h_{i j}(x) \mid x \in \Sigma\right\}
$$

The transition amplitude from the section $\Sigma_{i n}$ to the section $\Sigma_{f}$ is the integral over all geometries allowable by the boundary conditions:

$$
A_{f, i n}=\left\langle h_{f}, \Sigma_{f} \mid h_{i n}, \Sigma_{i n}\right\rangle=\int_{h_{i n}}^{h_{f}} D g \exp [i S(g)] .
$$

The absence of the Planck constant in the exponential function is a result of choosing the appropriate units of measurement. It will be shown below that the Planck constant $\hbar$ naturally emerges after the definition of the dimensional units simultaneously with gravitational constant $G$. However, the statement on the unification of gravity and quantum theory would be premature. The essence of quantum mechanics is based on the rule of summation of the transition amplitudes (5), which is postulated originally.

What is the probability of such a process? The answer is far from clarity even for the birth of a 4-dimensional space in the standard theory of gravitation linear in scalar curvature [22, 25]. As we need only to verify that this probability is nonzero, let us approximate the Largrangian by a linear theory. This is ensured by the condition (2). 
Action in the space $M_{D}$ is chosen in the form

$$
S_{D}=N_{0} \int d^{D} X \sqrt{\left|G^{(D)}\right|} F\left(R ; a_{n}\right) ; \quad F\left(R ; a_{n}\right)=\sum_{n} a_{n} R^{n}, \quad a_{1}=1,
$$

where $G^{(D)} \equiv \operatorname{det}\left(G_{A B}\right), R$ is a Ricci scalar, $N_{0}$ and $\left\{a_{i}\right\}$ are constants (see, for example, 10, 11, 12]). Note that normalization constant $N_{0}$ equals $1 /(16 \pi G)$ only in a low energy limit. Let us refer to $S_{D}$ as the first generation action. The standard form is $F\left(R ; a_{n}\right)=R-2 \Lambda$, i.e. $a_{1}=1, \quad a_{0}=-2 \Lambda$.

First step of a cascade begins in a small volume region with forming a subspace (11) with the metric of the form

$$
d s^{2}=G_{A B} d X^{A} d X^{B}=\mathrm{N} d t^{2}-g_{a b}(x) d x^{a} d x^{b}-e^{2 \beta(x)} \gamma_{i j}(y) d y^{i} d y^{j} .
$$

where $g_{a b}(x)$ is a spatial part of metric in $M_{D_{1}}, \gamma_{i j}(y)$ is a positively defined metric of the extra space $M_{d_{1}}$ and $e^{2 \beta(x)}$ is a scaling factor (see [16, 17]). Metric (77) is the generalisation of minisuperspace geometry, see e.g. [18]

$$
d s^{2}=\sigma^{2}\left[N(t)^{2} d t^{2}-a(t)^{2} d \Omega_{D_{1}}^{2}\right] .
$$

Here $N(t)$ is a lapse function, and $a(t)$ is a scale factor. The factor $\sigma^{2}$ is usually expressed as $\sigma^{2}=1 /\left(12 \pi^{2} M_{P l}^{2}\right)$ when dealing with 4-dimensional gravity.

Let us find the probability of producing such a space, approximating it by a linear dependence on $R$. Note that owing to the form of the chosen metric (7), the following relationships hold true (see [8]):

$$
\begin{aligned}
& R_{D}=R_{D_{1}}+R_{d_{1}}\left(\gamma_{i j}\right)+f_{\text {der }}, \\
& \phi \equiv R_{d_{1}}\left(\gamma_{i j}\right)=k d_{1}\left(d_{1}-1\right) e^{-2 \beta(x)} \\
& f_{\text {der }}=2 d_{1} g^{a b} \nabla_{a} \nabla_{b} \beta+d_{1}\left(d_{1}+1\right) g^{a b} \partial_{a} \beta \partial_{b} \beta,
\end{aligned}
$$

The covariant derivative $\nabla$ acts in the space $M_{D_{1}}$.

As was shown in 14, an extra space with an arbitrary geometry evolves into a space with a maximum number of Killing vectors for a given topology. So we can choose a maximally symmetric space with a constant Ricci scalar $R_{d_{1}}$ and a curvature $k= \pm 1$.

The volume $V_{d_{1}}$ of an internal space of a unit curvature depends on its geometry, because it is given by its intrinsic metric

$$
V_{d_{1}}=\int d^{d_{1}} y \sqrt{\left|G^{\left(d_{1}\right)}\right|}
$$

Let us emphasize that it would suffice to demand that only a small fracture of the volume of the initial space $M_{D}$ has the geometry (7). The following discussion is concerned with the point of view of an internal observer within $M_{D_{1}}$, who cannot get any information from the outside.

We will use the slow-change approximation proposed in [8]:

$$
\left|R_{d_{1}}\right| \gg\left|R_{D_{1}}\right|, \quad\left|R_{d_{1}}\right| \gg\left|f_{\text {der }}\right|
$$


Substituting Eq.(9) into Eq.(6) yields

$$
F\left(R_{D}\right)=F\left(R_{D_{1}}+R_{d_{1}}+f_{\text {der }}\right) \simeq F\left(R_{d_{1}}\right)+F^{\prime}\left(R_{d_{1}}\right) R_{D_{1}}+F^{\prime}\left(R_{d_{1}}\right) f_{\text {der }}
$$

Also, determinants satisfy

$$
\left|G^{(D)}\right|=e^{2 \beta(x)} \cdot\left|G^{\left(D_{1}\right)}\right| \cdot\left|G^{\left(d_{1}\right)}\right| .
$$

Substituting these expressions into action (6) and carrying out certain computations given explicitly in [8], we obtain a Lagrangian of a scalar-tensor gravitation in $D_{1}$ dimensions

$$
\begin{aligned}
& S_{D_{1}}=N_{0}^{\prime} \int d^{D_{1}} x \sqrt{\left|G^{\left(D_{1}\right)}\right|}\left(\operatorname{sign} F^{\prime}\right)\left[R_{D_{1}}+\frac{1}{2} K(\phi)(\partial \phi)^{2}-U(\phi)\right], \\
& K(\phi)=\frac{1}{2 \phi^{2}}\left[6 \phi^{2}\left(\frac{F\left(\phi ; a_{n}\right)^{\prime \prime}}{F\left(\phi ; a_{n}\right)^{\prime}}\right)^{2}-2 d_{1} \phi \frac{F\left(\phi ; a_{n}\right)^{\prime \prime}}{F\left(\phi ; a_{n}\right)^{\prime}}+\frac{1}{2} d_{1}\left(d_{1}+2\right)\right] \\
& U(\phi)=-\left(\operatorname{sign} F\left(\phi ; a_{n}\right)^{\prime}\right)\left[\frac{|\phi|}{d_{1}\left(d_{1}-1\right)}\right]^{d_{1} / 2} \frac{F\left(\phi ; a_{n}\right)}{F^{\prime}\left(\phi ; a_{n}\right)^{2}}
\end{aligned}
$$

In accordance with (11), we have kept only the term linear in Ricci scalar.

The quantum birth of the Universe in the linear theory has been studied by many authors. It is usually examined within the framework of minisuperspace, where the interval is written in the form (8)

The probability of the $D_{1}$-dimensional space quantum birth was calculated in [15. The universe creation probability in the presence of a scalar field has been studied in many papers, for instance [25]. In Vilenkin's approach, the probability of a 3-dimensional space birth is $d P \propto \exp \left[\frac{+2}{3 U(\phi)}\right]$, while the Hartle-Hawking approximation yields $d P \propto \exp \left[\frac{-2}{3 U(\phi)}\right]$. For all their differences, the main result of both approaches is a nonzero probability of such an event. Hence, the fraction of the universes with given properties produced by a cascade of reductions is nonzero.

\subsubsection{Stage of the classical evolution}

In order to estimate the probability of the quantum birth of a space consisting of two subspaces - the main one and the additional one - we restricted the discussion in previous section 2.2.1 to a linear in scalar curvature approximation.

Let us confine ourselves to those initial parameters, which lead to potentials having minima. Our numerical calculations indicate that such parameters do exist.

After nucleation, classical dynamics of these subspaces is as follows. A compact extra subspace evolves in such a way that the field $\phi$ approaches the value $\phi_{m}$, corresponding to the minimum of the potential $U(\phi)$.

It follows from the definition of the field $\phi$ (9) and the expression for the interval (77) that the characteristic size of the space $M_{d_{1}}$ is proportional to $e^{\beta} \propto$ 
$1 / \sqrt{\phi}$. Therefore, the size of a compact extra space quickly stabilizes when the field reaches the value $\phi=\phi_{m}$. This corresponds to $R_{d_{1}} \rightarrow$ const, $\quad f_{d e r} \rightarrow 0$. Therefore, in the following discussion we can use the conditions

$$
R_{d_{1}}=\text { const }, \quad f_{d e r}=0,
$$

which significantly simplify the calculations.

The equation for the scaling factor of the main subspace during de Sitter stage is of the form [16]

$$
\frac{D_{1}\left(D_{1}-1\right)}{2}(\dot{a} / a)^{2}=\Lambda-\frac{D_{1}\left(D_{1}-1\right)}{2} k
$$

We assume that the size of an extra space $M_{d_{1}}$ has stabilized and $U(\phi) \simeq$ $U\left(\phi_{m}\right) \equiv \Lambda$. Consequently, the scaling factor depends on time as

$$
a(t) \propto e^{H t}, \quad H=\frac{2 \Lambda}{D_{1}\left(D_{1}-1\right)}
$$

for large $t$. The size of a main subspace rapidly increases.

Thus, we have $D_{1}$-dimensional quickly expanding space and $d_{1}$-dimensional compact extra space. Linear in curvature approximation was sufficient to obtain this result, but to advance further we will need a more accurate expression for the reduced action. The latter could be derived using conditions (17). Expanding formula $F\left(R ; a_{n}\right)=F\left(R_{d_{1}}+R_{D_{1}} ; a_{n}\right)$ into Taylor series and integrating over coordinates of extra dimensions, we obtain

$$
S_{D_{1}}=N_{0}^{\prime} \int d^{D_{1}} X \sqrt{\left|G^{\left(D_{1}\right)}\right|} F\left(R_{D_{1}} ; \tilde{a}_{n}\right) .
$$

Here new parameters $\tilde{a}_{n}$ are functions of $R_{d_{1}}, n>0$.

We arrive at the second generation of action (19), which is similar to the first generation of action (6) with changed numerical values of the parameters $\tilde{a}_{n}$. Dimensionality of the main space has been reduced, $D_{1}<D$, and a new compact extra space of $d_{1}=D-D_{1}$ dimensions has been formed.

The size of the space $M_{D_{1}}$ is significantly larger than the size of $M_{d_{1}}$. In this paper we are concerned only with quantum fluctuations creating spaces satisfying such relation. Subsequent dynamics further increases their disparity.

The second step of a cascade is analogous to the first one with substitution $S_{D} \rightarrow S_{D_{1}}$ : in a small region of the space $M_{D_{1}}$ there occurs a quantum fluctuation which creates a subspace with topology $M_{D_{2}} \otimes M_{d_{2}} ; \quad D_{2}+d_{2}=D_{1}$. As a result of classical dynamics, the size of the space $M_{d_{2}}$ is stabilized and the space $M_{D_{2}}$ is expanding.

If we wish not to be concerned with excitations of the compact space $M_{d_{1}}$, we should only consider such quantum fluctuations in $M_{D_{1}}$ that satisfy $R_{d_{2}}<<$ $R_{d_{1}}$. 


\section{Quadratic gravity as an explicit example}

Initial action for gravitational field includes all powers of Ricci scalar and other invariants. A vast majority of the works on the subject uses some finite polynomial in Ricci scalar. A choice of a particular polynomial may be justified as follows. Let us consider a quantum fluctuation that produces a geometry with characteristic value of a scalar curvature $R_{0}$. Then the initial Lagrangian (6) may be approximated by a finite polynomial:

$$
F\left(R, a_{n}\right) \simeq \sum_{k=-K}^{K^{\prime}} b\left(R_{0}\right)_{k}\left(R-R_{0}\right)^{k}
$$

Specific values of $K$ and $K^{\prime}$ are designated according to the author's purposes. Coefficients $b_{k}\left(R_{0}\right)$ depend on the location of the expansion (20) and vary over a wide range.

Consider quadratic gravity in the space $M_{D}$ with an action of the form

$$
\begin{aligned}
S_{D}=\frac{N_{0}}{2} \int d^{D} X \sqrt{\left|G^{(D)}\right|}\left[R_{D}\left(G_{A B}\right)+C R_{D}^{2}\left(G_{A B}\right)+C_{1} R_{A B} R^{A B}+\right. \\
\left.+C_{2} \mathcal{K}-2 \Lambda\right]+\int_{\partial M_{D}} K d^{D-1} \Sigma,
\end{aligned}
$$

where we also included Ricci tensor and Kretschmann scalar $\mathcal{K}=R_{A B C D} R^{A B C D}$ The boundary term $\left(\partial M_{D}\right)$ introduced by Hawking and Gibbons does not influence classical dynamics and we ignore it in the following [15].

Let us try to find the values of the parameters that allow a universe similar to ours to form. In this case a set of parameters of the Lagrangian $\left\{a_{n}\right\}$ (see (6i)) is $\left\{C, C_{1}, C_{2}, \Lambda\right\}$.

Following the steps outlined in section 2.1, we will find the form of an action (21), reduced to space $M_{D_{1}}$. An action pertaining only to $M_{D_{1}}$ can be recovered by integrating action in $M_{D}$ (21) over $M_{d_{1}}$.

Ricci scalar can be expressed using equations (91):

$$
R_{D}\left(G_{A B}\right)=R_{D_{1}}\left(g_{a b}\right)+\phi(x)
$$

where we have set $f_{d e r}=0$ - see (17). The decomposition of $R_{A B} R^{A B}$ and $\mathcal{K}$ is given by (see [8])

$$
\begin{aligned}
R_{A B} R^{A B} & =R_{a b} R^{a b}+e^{-4 \beta(x)} \cdot R_{\mu \nu} R^{\mu \nu} \\
\mathcal{K} & =\mathcal{K}\left(g_{a b}\right)+e^{-4 \beta(x)} \cdot \mathcal{K}\left(\gamma_{i j}\right),
\end{aligned}
$$

where variables with indexes $A, B$ correspond to metric $G_{A B}$, those with indexes $a, b$ correspond to $g_{a b}$, and indexes $\mu, \nu$ correspond to metric $\gamma_{i j}$, (i.e. those values correspond to spaces $M_{D}, M_{D_{1}}$ and $M_{d_{1}}$ respectively). 
To advance further, recall that we are considering the $d_{1}$-dimensional space metric $\gamma_{i j}$ of a constant curvature $k$, so that we can express the Riemann tensor, Ricci tensor and Ricci scalar in $M_{d_{1}}$ space in terms of its curvature:

$$
R_{\rho \eta}^{\mu \nu}=k \delta_{\rho \eta}^{\mu \nu} ; \quad R_{\mu}^{\nu}=k\left(d_{1}-1\right) \delta_{\mu}^{\nu} ; \quad R_{d_{1}}^{\prime} \equiv k d_{1}\left(d_{1}-1\right)
$$

where $\delta_{\rho \eta}^{\mu \nu}=\delta_{\rho}^{\mu} \delta_{\eta}^{\nu}-\delta_{\eta}^{\mu} \delta_{\rho}^{\nu} . R_{d_{1}}^{\prime}$ represents the characteristic scale of curvature of extra dimensions. Expressions for squared Riemann and Ricci tensors are derived from (24); the former is by definition a Kretschmann scalar:

$$
\begin{gathered}
R_{\mu \nu} R^{\mu \nu}=d_{1}\left[k\left(d_{1}-1\right)\right]^{2} \\
\mathcal{K}\left(\gamma_{i j}\right) \equiv R_{\mu \nu \rho \eta} R^{\mu \nu \rho \eta}=2 d_{1}\left(d_{1}-1\right) k^{2}
\end{gathered}
$$

Now we can rewrite (23) substituting $e^{\beta(x)}$ from (9) and using (25):

$$
\begin{aligned}
& R_{A B} R^{A B}=R_{a b} R^{a b}+\frac{1}{d_{1}} \phi(x) \\
& \mathcal{K}=\mathcal{K}\left(g_{a b}\right)+\frac{2}{d_{1}\left(d_{1}-1\right)} \phi(x),
\end{aligned}
$$

After plugging (22) and (26) into action (21) and grouping the terms we obtain

$$
\begin{aligned}
S_{D}= & \frac{N_{0}}{2} \int d^{D} x \sqrt{\left|G^{(D)}\right|} \times\left\{R_{D_{1}}\left(g_{a b}\right)(1+2 C \phi)+C R_{D_{1}}^{2}\left(g_{a b}\right)-2 \Lambda+\right. \\
& \left.+C_{1} R_{a b} R^{a b}+C_{2} \mathcal{K}\left(g_{a b}\right)+\phi+\left(C+\frac{C_{1}}{d_{1}}+\frac{2 C_{2}}{d_{1}\left(d_{1}-1\right)}\right) \phi^{2}\right\}= \\
= & \frac{N_{0}}{2} \int d^{D} x \sqrt{\left|G^{(D)}\right|} \cdot \mathcal{L}\left(g_{a b}\right)
\end{aligned}
$$

The value in brackets is denoted $\mathcal{L}\left(g_{a b}\right)$ for convenience; it does not depend on the coordinates of extra space $M_{d_{1}}$.

To find the action in space $M_{D_{1}}$ we'll have to integrate (27) over the space $M_{d_{1}}$ using definition of the volume of that space (10). Substituting relationship (13) for $\sqrt{\left|G^{(D)}\right|}$ and relationships (9) and (24) for $e^{2 \beta(x)}$ yields:

$$
\begin{aligned}
S_{D} & =\frac{N_{0}}{2} \int d^{D} x \sqrt{\left|G^{(D)}\right|} \cdot \mathcal{L}\left(g_{a b}\right)= \\
& =\frac{N_{0}}{2} \int d^{D_{1}} x e^{2 \beta(x) d_{1}} \sqrt{\left|G^{\left(D_{1}\right)}\right|} \cdot \mathcal{L}\left(g_{a b}\right) \times \int d^{d_{1}} x \sqrt{\left|G^{\left(d_{1}\right)}\right|}= \\
& =\frac{N_{0} V_{d_{1}}}{2} \int d^{D_{1}} x \sqrt{\left|G^{\left(D_{1}\right)}\right|}\left(\frac{R_{d_{1}}^{\prime}\left(G^{\left(d_{1}\right)}\right)}{\phi(x)}\right)^{d_{1} / 2} \cdot \mathcal{L}\left(g_{a b}\right)
\end{aligned}
$$

Let us suppose that the minimum of a potential $U(\phi)$ exists. The field $\phi(x)$ rapidly relaxes to it and stays fixed during the low-energy processes (see 14 for discussion). This case is the most natural, since the relaxation time is 
proportional to the scale of the extra space $M_{d_{1}}$, which is small compared to the scale of the space $M_{D_{1}}$.

Assuming those conditions are satisfied, let us perform a conformal transformation of the form (see, for instance, 20]

$$
\begin{aligned}
& g_{a b}=\left|f\left(\phi_{m}\right)\right|^{-2 /\left(D_{1}-2\right)} \widetilde{g}_{a b}, \quad f(\phi) \equiv \phi^{-d_{1} / 2}(x)[1+2 C \phi(x)], \\
& R_{D_{1}}=\left|f\left(\phi_{m}\right)\right|^{2 /\left(D_{1}-2\right)} \widetilde{R}_{D_{1}}, \\
& \mathcal{K}=\left|f\left(\phi_{m}\right)\right|^{8 /\left(D_{1}-2\right)} \widetilde{\mathcal{K}} \\
& \sqrt{\left|G^{\left(D_{1}\right)}\right|}=\left|f\left(\phi_{m}\right)\right|^{-D_{1} /\left(D_{1}-2\right)} \sqrt{\left|\widetilde{G}^{\left(D_{1}\right)}\right|},
\end{aligned}
$$

which, being applied to Eq. (28), brings us to the initial form of the action (compare with (210):

$$
\begin{aligned}
S_{D_{1}}= & \frac{N_{0}^{1}}{2} \int d^{D_{1}} x \sqrt{\left|G^{\left(D_{1}\right)}\right|}\left\{R_{D_{1}}\left(g_{a b}\right)+C^{\left(D_{1}\right)} R_{D_{1}}\left(g_{a b}\right)^{2}+\right. \\
& \left.+C_{1}^{\left(D_{1}\right)} R_{a b} R^{a b}\left(g_{a b}\right)+C_{2}^{\left(D_{1}\right)} \mathcal{K}\left(g_{a b}\right)-2 \Lambda^{\left(D_{1}\right)}\right\},
\end{aligned}
$$

where the tildes were omitted for short. New parameters are expressed in terms of the old ones by the following equations:

$$
\begin{aligned}
C^{\left(D_{1}\right)}= & \operatorname{sign}\left(f\left(\phi_{m}\right)\right)\left|f\left(\phi_{m}\right)\right|^{\left(4-D_{1}\right) /\left(D_{1}-2\right)} \phi_{m}^{-d_{1} / 2} C, \\
\Lambda^{\left(D_{1}\right)}= & \operatorname{sign}\left(f\left(\phi_{m}\right)\right)\left|f\left(\phi_{m}\right)\right|^{-D_{1} /\left(D_{1}-2\right)} \phi_{m}^{-d_{1} / 2} \times \\
& \times\left[\Lambda-1 / 2\left(\phi_{m}+\left(C+\frac{C_{1}}{d_{1}}+\frac{2 C_{2}}{d_{1}\left(d_{1}-1\right)}\right) \phi_{m}^{2}\right)\right], \\
C_{1}^{\left(D_{1}\right)}= & \operatorname{sign}\left(f\left(\phi_{m}\right)\right)\left|f\left(\phi_{m}\right)\right|^{\left(4-D_{1}\right) /\left(D_{1}-2\right)} \phi_{m}^{-d_{1} / 2} C_{1}, \\
C_{2}^{\left(D_{1}\right)}= & \operatorname{sign}\left(f\left(\phi_{m}\right)\right)\left|f\left(\phi_{m}\right)\right|^{\left(8-D_{1}\right) /\left(D_{1}-2\right)} \phi_{m}^{-d_{1} / 2} C_{2} .
\end{aligned}
$$

where $f(\phi)$ is defined by (29). Recall that we are considering the case where the field $\phi$ is already at its minimum, $\phi=\phi_{m}$, so the kinetic terms were neglected. Equations (31)-(34) connect old and new parameters after one reduction.

Next reduction leads to analogous formulas for parameters $C^{\left(D_{2}\right)}, C_{1}^{\left(D_{2}\right)}$, $C_{2}^{\left(D_{2}\right)}, \Lambda^{\left(D_{2}\right)}$ with substitutions $C \rightarrow C^{\left(D_{1}\right)}, C_{1} \rightarrow C_{1}^{\left(D_{1}\right)}, C_{2} \rightarrow C_{2}^{\left(D_{1}\right)}, \Lambda \rightarrow$ $\Lambda^{\left(D_{1}\right)}$. Thus, we have obtained recurrence formulas for the parameters.

Action (30) for a subspace $M_{D_{1}}$ coincides in the form with the initial one (21) for $M_{D}$, but with renormalized parameters $C^{\left(D_{1}\right)}, C_{1}^{\left(D_{1}\right)}, C_{2}^{\left(D_{1}\right)}, \Lambda^{\left(D_{1}\right)}$.

\subsection{The final reduction. Formation of the low energy physics.}

It was shown in the previous section that the form of the action (21) does not change after space reductions (77). Now we shall consider the final reduction of an arbitrary cascade of $(n+1)$ reductions - the reduction leading to a fourdimensional space-time. 
It was shown in [8] that the action (21) may be reduced to the four-dimensional form

$$
\begin{gathered}
S \simeq \frac{N_{0} V_{d}}{2} \int d^{4} x \sqrt{\left|G^{(4)}\right|}\left[R_{4}+K(\phi)(\partial \phi)^{2}-2 U(\phi)\right] \\
U(\phi)=-\frac{1}{2} \operatorname{sign}\left(1+2 C^{\left(D_{n}\right)} \phi\right) \cdot\left[\frac{|\phi|}{d_{1}\left(d_{1}-1\right)}\right]^{d_{1} / 2} \frac{C_{t o t}^{\left(D_{n}\right)} \phi^{2}+\phi-2 \Lambda}{\left(1+2 C^{\left(D_{n}\right)} \phi\right)^{2}} \\
K(\phi)=\frac{\left(C^{\left(D_{n}\right)}\right)^{2} \phi^{2}\left(d_{1}^{2}-2 d_{1}+12\right)+C^{\left(D_{n}\right)} d_{1}^{2} \phi+\frac{1}{4} d_{1}\left(d_{1}+2\right)}{\left(1+2 C^{\left(D_{n}\right)} \phi\right)^{2} \phi^{2}}+ \\
\quad+\frac{C_{1}^{\left(D_{n}\right)}+C_{2}^{\left(D_{n}\right)}}{2 \phi\left(1+2 C^{\left(D_{n}\right)} \phi\right)} \\
\text { where } C_{\text {tot }}^{\left(D_{n}\right)} \equiv C^{\left(D_{n}\right)}+\frac{C_{1}^{\left(D_{n}\right)}}{d_{1}}+\frac{2 C_{2}^{\left(D_{n}\right)}}{d_{1}\left(d_{1}-1\right)}
\end{gathered}
$$

(compare with (15), (16), which are written for the case $C_{1}^{(D)}=C_{2}^{(D)}=0$ ). These equations, though derived for the action (21), are still applicable after any number of reductions, because the reductions do not change the form of the action, as was shown in the previous section.

In the vicinity of the minimum, $U\left(\phi_{m}\right) \equiv \min (U(\phi))$, potential can be expanded in a Taylor series so that the action (35) becomes

$$
\begin{gathered}
S \simeq \frac{N_{0} V_{d}}{2} \int d^{4} x \sqrt{\left|G^{(4)}\right|}\left[R_{4}+K\left(\phi_{m}\right)(\partial \phi)^{2}-2 U\left(\phi_{m}\right)-U^{\prime \prime}\left(\phi_{m}\right)\left(\phi-\phi_{m}\right)^{2}-\right. \\
\left.-\frac{1}{3} U^{\prime \prime \prime}\left(\phi_{m}\right)\left(\phi-\phi_{m}\right)^{3}-\ldots\right] .
\end{gathered}
$$

The unit of measurement I is still arbitrary. Let the units be related by expressions

$$
\mathrm{I}=\alpha \cdot \mathrm{cm},
$$

where $\alpha$ is as yet an unknown parameter. We change over to the standard units of length:

$$
\begin{gathered}
S=N_{0} V_{d} c \alpha^{2} \int d t d^{3} x\left[R_{4}+\frac{1}{2} K\left(\phi_{m}\right)(\partial \phi)^{2}-\alpha^{2} U\left(\phi_{m}\right)-\alpha^{2} \frac{1}{2} U^{\prime \prime}\left(\phi_{m}\right)\left(\phi-\phi_{m}\right)^{2}-\right. \\
\left.-\alpha^{2} \frac{1}{6} U^{\prime \prime \prime}\left(\phi_{m}\right)\left(\phi-\phi_{m}\right)^{3}\right] .
\end{gathered}
$$

Here, $x \rightarrow \alpha x, \sqrt{\left|G^{(4)}\right|}=c_{I}=\alpha c, R_{4} \rightarrow R_{4} / \alpha^{2}$ and $(\partial \phi)^{2} \rightarrow(\partial \phi)^{2} / \alpha^{2}$.

Since expression (40) is the low-energy limit of action (21), it should adequately describe purely gravitational phenomena. Moreover, expression (40) makes it possible to explain the origin of the inflaton potential and the cosmological constant. The effective action (40) contains the initial parameters of the theory without involving fundamental constants, such as the Planck constant $\hbar$ 
and the gravitational constant $G$. Now we will find the relations between those values.

Let us introduce the definition

$$
m_{\Phi} \equiv \alpha \sqrt{\frac{U^{\prime \prime}\left(\phi_{m}\right)}{K\left(\phi_{m}\right)}}
$$

and, under the assumption $K\left(\phi_{m}\right)>0$, introduce the variable

$$
\Phi=\sqrt{\frac{c^{4}}{16 \pi G} K\left(\phi_{m}\right)}\left(\phi-\phi_{m}\right) .
$$

The action (40) in the terms of $\Phi$ is

$$
\begin{gathered}
S=\frac{16 \pi G}{c^{4}} N_{0} V_{d} c \alpha^{2} \int d t d^{3} x\left[\frac{c^{4}}{16 \pi G} R_{4}+\frac{1}{2}(\partial \Phi)^{2}-\frac{c^{4}}{16 \pi G} \alpha^{2} U\left(\phi_{m}\right)-\frac{1}{2} m_{\Phi}^{2} \Phi^{2}\right. \\
\left.-\frac{1}{6} \sqrt{\frac{16 \pi G}{c^{4}}} m_{\Phi}^{2} \frac{U^{\prime \prime \prime}\left(\phi_{m}\right)}{U^{\prime \prime}\left(\phi_{m}\right) \sqrt{K\left(\phi_{m}\right)}} \Phi^{3}\right]
\end{gathered}
$$

We arrive at the conventional form of the action for the scalar field $\Phi$ interacting with gravity:

$$
S=\frac{1}{\hbar} \int d t d^{3} x\left(\frac{c^{4}}{16 \pi G} R_{4}+\frac{1}{2}(\partial \Phi)^{2}-\Lambda-\frac{1}{2} m_{\Phi}^{2} \Phi^{2}+\lambda_{3} \Phi^{3}\right) .
$$

where $G$ is the gravitational constant, $c$ is the speed of light, $\hbar$ is the Planck's constant, $\Lambda$ is the dark energy density and $m_{\Phi}$ is the mass of the scalar field which we associate with inflaton.

Comparing (43) with (42) we obtain the relations

$$
\begin{aligned}
& \frac{1}{\hbar}=\frac{16 \pi G}{c^{3}} N_{0} V_{d} \alpha^{2} \\
& \Lambda=\frac{c^{4}}{16 \pi G} \alpha^{2} U\left(\phi_{m}\right) \\
& \lambda_{3}=-\frac{1}{6} \sqrt{\frac{16 \pi G}{c^{4}}} m_{\Phi}^{2} \frac{U^{\prime \prime \prime}\left(\phi_{m}\right)}{U^{\prime \prime}\left(\phi_{m}\right) \sqrt{K\left(\phi_{m}\right)}}
\end{aligned}
$$

By eliminating the parameter $\alpha$ from relationships (44), (45), (46) and (41), we obtain

$$
\begin{aligned}
\frac{U\left(\phi_{m}\right) K\left(\phi_{m}\right)}{U^{\prime \prime}\left(\phi_{m}\right)} & =\frac{16 \pi G}{c^{4}} \frac{\Lambda}{m_{\Phi}^{2}}, \\
N_{0} V_{d} \frac{K\left(\phi_{m}\right)}{U^{\prime \prime}\left(\phi_{m}\right)} & =\frac{c^{3}}{16 \pi G \hbar m_{\Phi}^{2}}, \\
\frac{U^{\prime \prime \prime}\left(\phi_{m}\right)}{U^{\prime \prime}\left(\phi_{m}\right) \sqrt{K\left(\phi_{m}\right)}} & =-6 \sqrt{\frac{c^{4}}{16 \pi G}} \frac{\lambda_{3}}{m_{\Phi}^{2}} .
\end{aligned}
$$


Right sides of equations (47)-(48) contain fundamental constants while the left ones depend on the initial parameters. If the field $\Phi$ is associated with the inflaton, its mass is about $10^{13} \mathrm{GeV}$. Parameter $\lambda_{3}$ defined in (46), is not yet measured and may be considered to be the prediction of our approach. During numerical calculations we kept in mind that $\lambda_{3}<10^{-12} M_{P l}$. It provides inflation, which does not contradict the observational data.

The conversion factor between the units of length $\alpha=\mathrm{I} / \mathrm{cm}$ can also be found by another method. Indeed, the characteristic size of the extra space is determined to be $V_{d}^{1 / d}$ in units of I. Moreover, by designating the size of the hypothetical extra space as $L_{d}$ expressed in terms of $\mathrm{cm}$, we obtain $\alpha=$ $L_{d} / V_{d}^{1 / d}$. Values of $L_{d} \leq 10^{-17}$ do not contradict experimental data. Taking into account expression (41), we find the constraint on the initial parameters

$$
L_{d}=m_{\Phi} V_{d}^{1 / d} \sqrt{\frac{K\left(\phi_{m}\right)}{U^{\prime \prime}\left(\phi_{m}\right)}}<10^{-17} .
$$

Relationships (47)-(50) allow us to compare the initial parameters of the theory - $N_{0}, C, C_{1}, C_{2}, \Lambda$ from the action (21) with observed constants $c, \hbar, G, \Lambda, m_{\Phi}$ and to predict the value of inflaton coupling constant $\lambda_{3}$. The question is whether we can find the initial parameters to satisfy the constraints (47)-(50). We have proved the existence of such parameters by numerical simulations of reduction cascades. As it is shown in the next section, we have found numerous sets of initial parameters satisfying those conditions.

\subsection{Numerical computations}

The numerical simulations are carried out in the following manner: first, we assign random values to initial parameters of cascade $-C, C_{1}, C_{2}, \Lambda$. Then we choose a cascade by specifying a sequence of dimensions of hyperspaces undergoing reductions, which should end with a four-dimensional space-time. For example, $\langle 15 \rightarrow 11 \rightarrow 8 \rightarrow 4\rangle$ constitutes a cascade going from a 15dimensional space to a 4-dimensional one. Then we calculate the values of the final parameters using recurrence formulas (31)-(34); substituting them into (36), (37) we obtain kinetic and potential terms before the last reduction. Those final parameters which satisfy the conditions (47)-(50), correspond to observable low-energy physics.

A set of initial parameters $\left\{C, C_{1}, C_{2}, \Lambda\right\}$ is represented by a point in the parametric hyperspace $\left(C, C_{1}, C_{2}, \Lambda\right)$. We shall refer to those parameter sets that lead to observable constants (i.e. satisfy (47)-(50) as solutions. We are interested in the density of solutions in the parametric hyperspace. To assess the density visually we have to use its plane projections - for instance projections on planes $\left(C_{1}, C_{2}\right)$ or $(C, \Lambda)$. Fig. 1 presents the results of numerical computation - projections of a four-dimensional solution image on planes $\left(C, C_{1}\right)$ and $(C, \Lambda)$.

It could be seen that there are numerous solutions. This means that the observed low-energy physics is reproduced in a wide range of initial parameters 


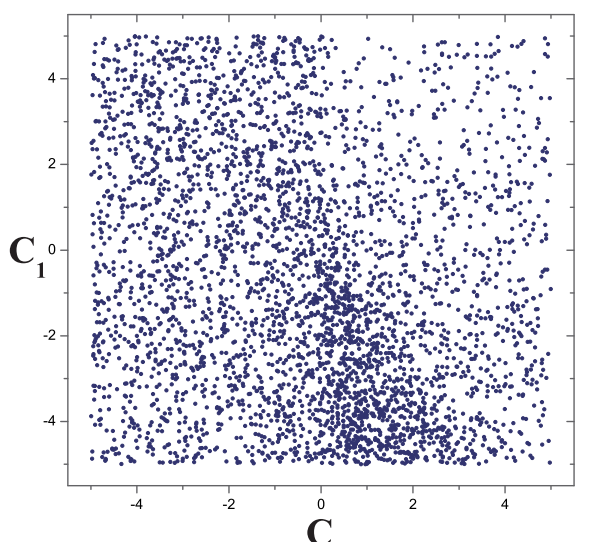

a)

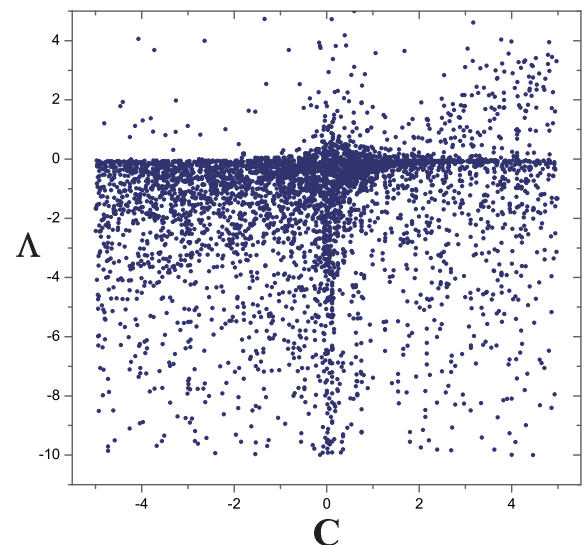

b)

Figure 1: Results of numerical computation: plane projections of the points in the hyperspace $\left(C, C_{1}, C_{2}, \Lambda\right)$ corresponding to solutions:

a) a projection on the plane $\left(C, C_{1}\right), \quad$ b) a projection on the plane $(C, \Lambda)$

of our theory.

Recall that we took into account only the most simple geometries of the hyperspaces undergoing reductions - only the maximally symmetrical ones. Including other geometries into consideration would yield additional solutions. If these solutions form a continuum in the parametric space, any point in that space (i.e. any set of initial parameters) would lead to the observable physics.

\section{Discussion}

The prospective theory, called "the Theory of Everything" (TOE), should solve the problem of the fine tuning of the universe. How are the initial parameters of TOE chosen and why do they allow very complex structures to arise? A way to approach this problem was found by proposing an existence of numerous vacua with different properties - a "landscape" in the modern terminology. The landscape, derived in the scope of the string theory, is an important advancement, but the questions to be answered still remain. Is the concept of 'strings' indispensable or will the assumption of multiplicity of dimensions alone suffice? How crucial are the values of the initial parameters? What is the probability of getting to a particular vacuum? Which additional values besides the metric do we need to include?

Within the framework of the theory derived in this paper these questions may be answered as follows:

- For the low-energy 'landscape' to exist we need only to assume the existence of multiple dimensions. The rest of the string theory tools as well 
as incorporation of additional fields are not necessary. The cascade reduction mechanism effectively produces the low-energy physics with various parameters.

- A variation of the initial parameters does not affect significantly the probability of a universe formation.

- Values of the Plank constant and the gravitational constant differ in different universes and depend on the choice of a reduction cascade for a given set of initial parameters. Those constants are changing with time during the early stages of the universe formation.

It has been shown that there are numerous values of initial parameters of the theory which could be "connected by a cascade" with observed fundamental constants. Particular numerical values of initial parameters are therefore not as important as it was previously thought.

The 'landscape' concept implies that numerous low-energy theories with various properties originate from a theory with unique initial parameters. This paper is concerned with an opposite scenario when the observed physics is derived from numerous initial theories. This 'inverted landscape' model brings into question the significance of a search for the unique Lagrangian of TOE.

A set of all such values is rather large, although does not constitute a continuum in the parametric hyperspace. This may be attributed to the limited subset of all possible cascades that we have studied - only those composed of absolutely symmetric compact spaces. Evidently an extension of this subset to include all possible geometries will increase the number of acceptable parameters.

\section{Conjecture}

For any given set of initial parameters there exists a cascade of reductions leading from a multidimensional space to a universe of our type.

The credibility of this hypothesis will be fully examined in the future research.

\section{Acknowledgements.}

We are grateful to K.A. Bronnikov for helpful discussions. This work was supported in part by the Russian Foundation for Basic Research (project no. 0902-00677-a) 


\section{References}

[1] J. D. Barrow, F. J. Tipler, The Anthropic Cosmological Principle, Clarendon Press, Oxford (1986).

M. J. Rees, Our Cosmic Habitat, Princeton Univ. Press, Princeton (2002).

N. Bostrom, Anthropic Bias: Observation Selection Effects in Science and Philosophy, Routledge, New York (2002).

M. Tegmark, Parallel Universes, "Science and Ultimate Reality: From Quantum to Cosmos", Cambridge University Press (2003), astro-ph/0302131, 2003;

A. Aguirre and M. Tegmark, JCAP 0501:003,2005, hep-th/0409072, 2004.

G. L. Kane, M. J. Perry, A. N. Zytkow, New Astron., 7, 45 (2002).

P. Danies, Mod.Phys.Lett. A, 19, 727 (2004).

C. J. Hogan, In Universe or Multiverse?, ed. by B.J.Carr, Cambridge University Press (2005), E-print archives, astro-ph/0407086.

W. R. Stoeger, G. F. R Ellis, and U. Kirchner, E-print archives, astro-ph/0407329.

A. Aguirre, In Universe or Multiverse, ed. by B. Carr, Cambridge University Press (2005), E-print archives, astro-ph/0506519.

M. Livio, M. J. Rees, Science, 309, 1022 (2005).

S. Weinstein, Class.Quant.Grav., 23, 4231 (2006), E-print archives, hep-th/0508006.

M. Tegmark et. al, Phys. Rev. D 73, 023505 (2006).

[2] W. Lerche, D. Lust and A. N. Schellekens, Nucl. Phys. B 287, 477 (1987).

[3] S.G. Rubin, in Chaos Solitons Fractals 14, 891, (2002); astro-ph/0207013,

S.G. Rubin, G\& C 9, 243, (2003); E-print archives, hep-ph/0309184.

[4] S. Kachru, R. Kallosh, A. Linde and S. P. Trivedi, Phys. Rev. D 68, 046005 (2003).

[5] C. Castro, A. Granik, M.S. El Naschie, E-print archives, hep-th/0004152.

[6] U. Bleyer, M. Mohazzab, M. Rainer, E-print archives, gr-qc/9508035

[7] R. Bousso and J. Polchinski, JHEP 0006, 006 (2000)

[8] K. A. Bronnikov and S. G. Rubin, Phys. Rev. D 73, 124019 (2006); E-print archives, gr-qc/0510107.

[9] S. G. Rubin, Zh. Eksp. Teor. Fiz. 133 (4), 820 (2008) [JETP 106 (4), 714 (2008)]; arXiv:0804.4348.

[10] A. A. Starobinsky, JETP Lett. 86, 157 (2007). 
[11] S. Nojiri, Sergei D. Odintsov, Int.J.Geom.Meth.Mod.Phys. 4, 115 (2007); arXiv:hep-th/0601213

[12] L. M. Sokolowski, Acta Phys. Polon., B 39, 2879 (2008).

[13] L. Susskind, arXiv hep-th/0302219

[14] S.G. Rubin. On the Origin of Gauge Symmetries and Fundamental Constants JETP 109, 961 (2009).

[15] S. Carlip, arXivigr-qc/0508072

[16] S. M. Carroll et al., Phys.Rev. D 66, 024036 (2002); arXiv:hep-th/0110149

[17] K. A. Bronnikov, R. V. Konoplich and S. G. Rubin, Class. Quantum Grav. 24, 1261 (2007); arXiv:gr-qc/0610003

[18] A. Vilenkin, Phys. Rev. D 37, 888, (1988).

[19] G.E. Volovik, Proceedings of "From Quantum to Emergent Gravity: Theory and Phenomenology" Phenomenology, Trieste, Italy, 2007 (Trieste, 2007), $\mathrm{PoS}(\mathrm{QG}-\mathrm{Ph})$

[20] K.A. Bronnikov and V.N. Melnikov, E-print archives, gr-qc/0310112.

[21] M. Blagojevic, Gravitation and gauge symmetries (Institute of Physics, Bristol, 2002).

[22] J.B. Hartle and S.W. Hawking, Phys. Rev. D 28, 2960 (1983).

[23] H. Ochiai, K. Sato, arXiv:gr-qc/0007059

[24] H. Firouzjahi, S. Sarangi and S.-H. Henry Tye, arXiv hep-th/0406107 2004

[25] A. Vilenkin, Phys. Lett. B 117, 25 (1982).

[26] D.L. Wiltshire, in Cosmology: the Physics of the Universe, Ed. by B. Robson, N. Visvanathan and W.S. Woolcock (World Scientific, Singapore, 1996), p. 473.

[27] M. J. Duff, L. B. Okun, G. Veneziano, JHEP 03, 023, 023 (2002), arxiv: physics/0110060.

[28] G. E. Volovik, arXiv 0904.1965 [gr-qc]

[29] T. A. Brun, J. B. Hartle, Phys.Rev. E 59, 6370 (1999); arXiv:quant-ph/9808024

[30] A. B. Katok and B.Hasselblatt, Introduction to the Modern Theory of Dynamical Systems (Cambridge University Press, Cambridge, 1996; Faktorial, Moscow, 1999). 
[31] G. D. Starkman, D. Stojkovic and M. Trodden, Phys.Rev. D 63, 103511 (2001); arXiv hep-th/0012226v2.

[32] B. A. Dubrovin, A. T. Fomenko, and S. P. Novikov, Modern Geometry: Methods and Applications (Nauka, Moscow 1979; Springer, New York, 1985).

[33] U. Günther, P. Moniz and A. Zhuk, Astrophys. Space Sci. 283, 679 (2003); gr-qc/0209045.

[34] I. Antoniadis, K. Benakli, Phys.Lett. B 331, 313 (1994); arXiv:hep-ph/9403290.

[35] H. Davoudiasl and T. G. Rizzo, Phys.Rev. D 76, 055009, (2007); arXiv hep-ph/0702078.

[36] M. Regis, M. Serone and P. Ullio, Phys.Lett. B 663, 250 (2008); hep-ph/0612286.

[37] J. Garriga, A. Vilenkin, Phys.Rev. D 64, 023517 (2001); e-Print: hep-th/0011262.

[38] A. D. Dolgov, F. R. Urban, Phys.Rev. D 77, 083503 (2008); arXiv:0801.3090

[39] D. H. Lyth and A. Riotto, Phys.Rept. 314, 1 (1999); arXiv:hep-ph/9807278v4 